\title{
Движение простой модели автомобиля
}

\author{
У. Боттема
}

В настоящей работе рассматривается движение плоской механической системы с четырьмя степенями свободы. Система движется в горизонтальной плоскости в отсутствие внешних сил. Она состоит из двух шарнирно связанных твердых тел $L_{1}$ и $L_{2}$, на каждое из которых наложены неголономные связи. Кинематическое исследование показывает, что нужно различать два основных случая: траектория средней точки $Q$ «задней оси» имеет или бесконечно много точек возврата и точек перегиба, или одну точку возврата и ни одной точки перегиба. Уравнения движения можно всегда элементарно проинтегрировать так, чтобы получить зависимость углов вращения тел от времени. Для специального случая удалось определить траекторию точки $Q$, которая является круговой трактрисой.

1. Рассмотрим движение плоской системы, схематично изображенной на рисунке 1. Система состоит из двух твердых тел $L_{1}$ и $L_{2}$, которые связаны друг с другом в точке $P$ с помощью шарнира. Прямая $P Q$ служит осью симметрии для тела $L_{1}$; расстояние $P Q$ равно $l$. Точка $S$ обозначает центр тяжести тела $L_{1}$, причем $Q S=a$. Масса тела $L_{1}$ равна $m_{1}$, а момент инерции в точке $S$ равен $m_{1} \rho_{1}^{2}$. Тело $L_{2}$ симметрично относительно точки $P$, его масса равна $m_{2}$, а момент инерции в точке $P$ равен $m_{2} \rho_{2}^{2}$. На систему накладывается условие, состоящее в том, что ни задняя ось $Q_{1} Q_{2}$, жестко связанная с телом $L_{1}$, ни передняя ось $P_{1} P_{2}$ не могут смещаться вдоль себя самих; кроме того, мы предполагаем, что на систему не действуют никакие внешние силы за исключением неизбежно возникающих сил трения. Такую систему можно рассматривать как модель автомобиля. При отсутствии тела $L_{2}$ мы получим сани Каратеодори [1].

2. Данная система имеет четыре степени свободы. Выберем в качестве координат прямоугольные координаты $x$ и $y$ для точки $Q$, угол $\vartheta$ между лучом $Q P$ и осью $X$, а также угол $\varphi$ между прямыми $Q P$ и $P P_{1}$. Тогда «естественным» положением автомобиля считается его положение при $\varphi=\pi / 2$ (или $\varphi=-\pi / 2)$. Точка $P$ имеет координаты

$$
x_{p}=x+l \cos \vartheta, \quad y_{p}=y+l \sin \vartheta .
$$

Bottema O. Die Bewegung eines einfachen Wagenmodells // Z. Angew. Math. Mech., 1964, vol. 44, no. 12, pp. 585-593.

Перевод с немецкого В. В. Шуликовской, под ред. А. А. Килина

НЕЛИНЕЙНАЯ ДИНАМИКА. 2015. Т. 11. № 3. С. 621-632 


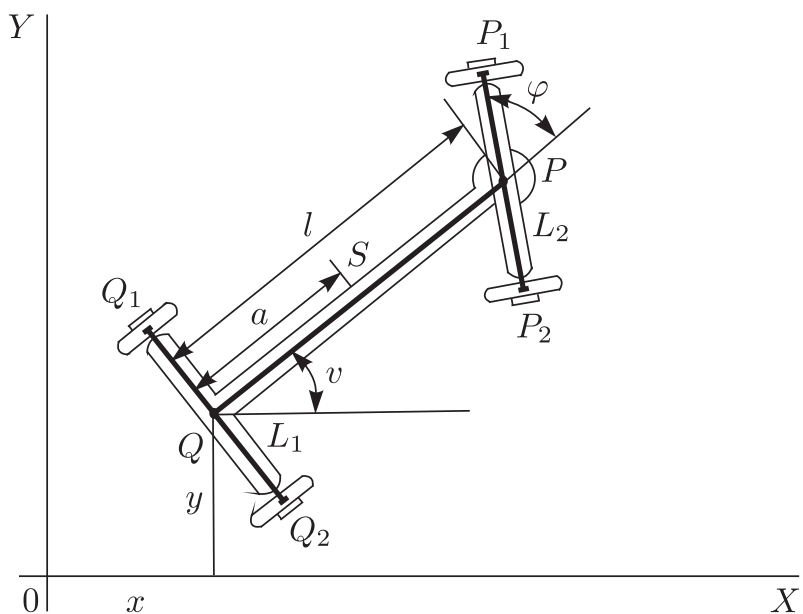

Рис. 1

Приравнивая к нулю скорости точек $Q$ и $P$ вдоль $Q_{1} Q_{2}$ и $P_{1} P_{2}$ соответственно, получаем неголономные кинематические условия

$$
\begin{gathered}
\dot{x} \sin \vartheta-\dot{y} \cos \vartheta=0, \\
\dot{x} \cos (\vartheta+\varphi)+\dot{y} \sin (\vartheta+\varphi)+l \dot{\vartheta} \sin \varphi=0,
\end{gathered}
$$

из которых следует, что $\dot{x}, \dot{y}$ и $\dot{\vartheta}$ связаны соотношением

$$
\dot{x}: \dot{y}: l \dot{\vartheta}=\cos \vartheta: \sin \vartheta:(-\operatorname{ctg} \varphi) .
$$

Если обозначить скорость точки $Q$ через $u$, то

$$
u=\dot{x} \cos \vartheta+\dot{y} \sin \vartheta, \quad \dot{x}=u \cos \vartheta, \quad \dot{y}=u \sin \vartheta, \quad l \dot{\vartheta}=-u \operatorname{ctg} \varphi
$$

3. Поскольку $x_{s}=x+a \cos \vartheta, y_{s}=y+a \sin \vartheta$, мы можем найти кинетическую энергию $T_{1}$ тела $L_{1}$,

$$
T_{1}=\frac{1}{2} m_{1}\left\{\dot{x}^{2}+\dot{y}^{2}-2 a \sin \vartheta \cdot \dot{x} \dot{\vartheta}+2 a \cos \vartheta \cdot \dot{y} \cdot \dot{\vartheta}+\left(\rho_{1}^{2}+a^{2}\right) \dot{\vartheta}^{2}\right\},
$$

тогда как кинетическая энергия тела $L_{2}$ равна

$$
T_{2}=\frac{1}{2} m_{2}\left\{\dot{x}^{2}+\dot{y}^{2}-2 l \sin \vartheta \cdot \dot{x} \dot{\vartheta}+2 l \cos \vartheta \cdot \dot{y} \dot{\vartheta}+l^{2} \dot{\vartheta}^{2}+\rho_{2}^{2}(\dot{\vartheta}+\dot{\varphi})^{2}\right\} .
$$

Общая кинетическая энергия системы составляет $T=T_{1}+T_{2}$, и, вспоминая о том, что потенциальная энергия равна нулю, мы можем воспользоваться кинематическими условиями (1) и $(2)$, чтобы записать четыре уравнения Лагранжа второго рода. Для $x$ и, соответственно, $y$ находим

$$
\left\{\begin{array}{l}
\frac{d}{d t}\left\{\left(m_{1}+m_{2}\right) \dot{x}-\left(m_{1} a+m_{2} l\right) \sin \vartheta \cdot \dot{\vartheta}\right\}=\lambda_{1} \sin \vartheta+\lambda_{2} \cos (\vartheta+\varphi), \\
\frac{d}{d t}\left\{\left(m_{1}+m_{2}\right) \dot{y}+\left(m_{1} a+m_{2} l\right) \cos \vartheta \cdot \dot{\vartheta}\right\}=-\lambda_{1} \cos \vartheta+\lambda_{2} \sin (\vartheta+\varphi)
\end{array}\right.
$$

с множителями $\lambda_{1}$ и $\lambda_{2}$, которые задают силы трения. 
Уравнение Лагранжа для переменной $\varphi$ принимает простой вид

$$
\dot{\vartheta}+\dot{\varphi}=c_{1}
$$

где $c_{1}$ - некоторая константа. Впрочем, данное уравнение вытекает из того факта, что силы, действующие на тело $L_{2}$, проходят через центр тяжести $P$.

Если теперь вместо уравнения Лагранжа на $\vartheta$ взять интеграл энергии

$$
T_{1}+T_{2}=\frac{1}{2} h_{1}^{2}
$$

(силы трения, очевидно, никакой работы не совершают), то формулы $(1),(2),(7)$ и (8) дадут нам систему уравнений движения, не содержащую $\lambda_{1}$ и $\lambda_{2}$. Из (5) и (7) получаем

$$
T_{2}=\frac{1}{2} m_{2}\left(\dot{x}^{2}+\dot{y}^{2}-2 l \sin \vartheta \cdot \dot{x} \dot{\vartheta}+2 l \cos \vartheta \cdot \dot{y} \dot{\vartheta}+l^{2} \dot{\vartheta}^{2}\right)+\frac{1}{2} m_{2} \rho_{2}^{2} c_{1}^{2} .
$$

Далее, воспользовавшись соотношениями (3), приходим к формулам

$$
\begin{aligned}
& T_{1}=\frac{1}{2} m_{1} u^{2}\left\{1+\frac{\rho_{1}^{2}+a^{2}}{l^{2}} \operatorname{ctg}^{2} \varphi\right\}, \\
& T_{2}=\frac{1}{2} m_{2} u^{2}\left\{1+\operatorname{ctg}^{2} \varphi\right\}+\frac{1}{2} m_{2} \rho_{2}^{2} c_{1}^{2} .
\end{aligned}
$$

Кроме того, введем положительную константу $h_{2}$ из равенства

$$
h_{2}^{2}=h_{1}^{2}-m_{2} \rho_{2}^{2} c_{1}^{2}
$$

так что в итоге $h_{2}^{2} / 2$ обозначает постоянную кинетическую энергию системы за вычетом постоянной энергии вращения тела $L_{2}$. В результате получим

$$
u^{2}\left(A+B \operatorname{ctg}^{2} \varphi\right)=h_{2}^{2},
$$

причем

$$
A=m_{1}+m_{2}, \quad B=m_{1} \frac{\rho_{1}^{2}+a^{2}}{l^{2}}+m_{2}
$$

- это положительные величины, характеризующие нашу систему. Теперь введем безразмерную положительную константу $k^{2}$ :

$$
k^{2}=\frac{B}{A}
$$

кроме того, положим $h_{2}^{2}=A l^{2} k^{2} c_{2}^{2}$, получая в итоге

$$
\frac{u}{l}=\frac{k c_{2} \sin \varphi}{\sqrt{\sin ^{2} \varphi+k^{2} \cos ^{2} \varphi}}, \quad \dot{\vartheta}=\frac{-k c_{2} \cos \varphi}{\sqrt{\sin ^{2} \varphi+k^{2} \cos ^{2} \varphi}} .
$$

Поскольку $u$ и $\dot{\vartheta}$ должны представлять собой непрерывные функции от $\varphi$, которые не обращаются в нуль одновременно, знак, стоящий перед квадратным корнем, должен быть одним и тем же в течение всего движения. Вспоминая о том, что у нас еще есть возможность выбрать знак константы $c_{2}$ по своему усмотрению, будем считать, что в формулах (14) знаменатель всегда больше нуля. Кроме того, мы можем считать, что и $k$ больше нуля. 
Тогда из (7) вытекает следующее дифференциальное уравнение первого порядка для $\varphi$ :

$$
\dot{\varphi}=c_{1}+\frac{k c_{2} \cos \varphi}{\sqrt{\sin ^{2} \varphi+k^{2} \cos ^{2} \varphi}},
$$

где $c_{1}$ и $c_{2}$ представляют собой константы интегрирования, имеющие размерность угловой скорости. Их можно определить из начальных условий. Если при $t=0$ у нас $\varphi=\varphi_{0}, \dot{\varphi}=\dot{\varphi}_{0}$ и $u=u_{0}$ (причем очевидно, что мы можем взять $x=y=\vartheta=0$ ), то получаем

$$
c_{1}=\dot{\varphi}_{0}+\frac{u_{0}}{l} \operatorname{tg} \varphi_{0}, \quad c_{2}=\frac{u_{0}}{k l \sin \varphi_{0}} \sqrt{\sin ^{2} \varphi_{0}+k^{2} \cos ^{2} \varphi_{0}} .
$$

4. Для того чтобы проинтегрировать (15), положим $\operatorname{tg} \varphi=k \operatorname{tg} \psi$. Тогда из (14) находим

$$
u=l k c_{2} \sin \psi, \quad \dot{\vartheta}=-c_{2} \cos \psi,
$$

из $(15)-$

$$
k \dot{\psi}=\left(k^{2} \sin ^{2} \psi+\cos ^{2} \psi\right)\left(c_{1}+c_{2} \cos \psi\right)
$$

а отсюда при $v=\operatorname{tg}(\psi / 2)$ следует

$$
2 k \dot{v}=\frac{\left\{v^{4}+2\left(2 k^{2}-1\right) v^{2}+1\right\}\left\{\left(c_{1}-c_{2}\right) v^{2}+\left(c_{1}+c_{2}\right)\right\}}{\left(1+v^{2}\right)^{2}} .
$$

В результате мы сможем выразить соотношение, связывающее $v$ и $t$, а следовательно, $\varphi$ и $t$, с помощью простой квадратуры (рационального интеграла). Вид этой квадратуры, кроме всего прочего, будет зависеть еще и от того, положительна или отрицательна разность $\left(c_{1}^{2}-c_{2}^{2}\right)$. Прежде чем мы перейдем к интегрированию уравнения (18), поясним, в чем состоит механический смысл этих трех разных случаев. Мы будем исходить из простых кинематических соображений.

5. У двух точек твердого тела $L_{1}$ направление скорости определено: скорость точки $Q$ направлена вдоль прямой $Q P$, а скорость точки $P$ перпендикулярна прямой $P_{1} P_{2}$. Отсюда следует, что точка $G$, в которой прямая $P_{1} P_{2}$ пересекает $Q_{1} Q_{2}$, служит мгновенным полюсом движения (рис. 2). Полярный луч $Q G$ равен $l \operatorname{tg} \varphi$ и, поскольку $\dot{\vartheta}$ представляет собой мгновенную угловую скорость, справедливо уже известное нам равенство $u=-l \dot{\vartheta} \operatorname{tg} \varphi$. Очевидно, что прямая $p=Q_{1} Q_{2}$ - это полярная кривая подвижной плоскости, причем она касается неподвижной полярной кривой в точке $G$; таким образом, прямая $p$ - это полярная касательная к траектории, и по известному закону кинематики точка $G$ совпадает с центром кривизны траектории $C(Q)$, описываемой точкой $Q$. Следовательно, радиус кривизны траектории $C(Q)$ равен $l|\operatorname{tg} \varphi|$. При движении точка $G$ перемещается в подвижной плоскости вдоль прямой $p$ с переменной полярной скоростью $\omega=l \dot{\varphi} / \cos ^{2} \varphi$, если положительное направление прямой $p$ задается лучом $Q_{1} Q_{2}$. Траектория поворота касается прямой $p$ в точке $G$; диаметр окружности поворота $2 R=\omega / \dot{\vartheta}$.

При $\varphi= \pm \pi / 2$ точка $G$ уходит в бесконечность, а движение представляет собой мгновенное поступательное движение; из (14) следует, что $\dot{\vartheta}=0$, а скорость поступательного движения равна $u= \pm k l c_{2}$. Траектория $C(Q)$ проходит через точку перегиба.

Еще два особых положения получаем при $\varphi=0$ и $\varphi=\pi$; тогда точка $G$ совпадает с $Q$, скорость $u$ равна нулю, а $\dot{\vartheta}=\mp c_{2}$. В этом положении траектория $C(Q)$ проходит через точку возврата. 


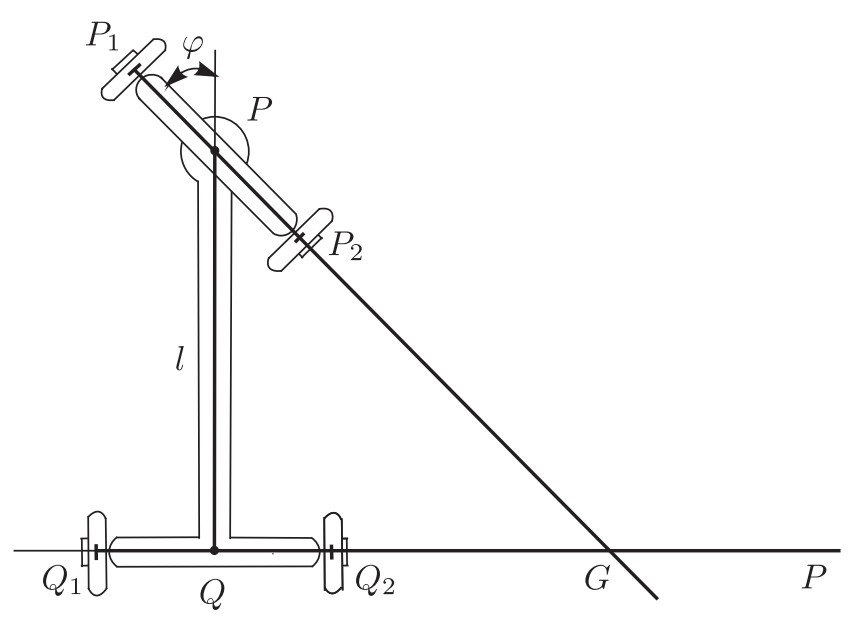

Рис. 2

Из всех этих рассуждений следует, что для движения очень существенно, совершает ли тело $L_{2}$ полный оборот вокруг точки $P$. Полный оборот совершается всегда, когда величина $\dot{\varphi}$ сохраняет один и тот же знак; в таком случае у траектории $C(Q)$ будет бесконечно много точек возврата и бесконечно много точек перегиба, чередующихся друг с другом на кривой.

Однако если бы в каком-то конкретном положении $\dot{\varphi}=0$, то в нуль обращалась бы и величина $\ddot{\varphi}=(d \dot{\varphi} / d \varphi) \dot{\varphi}$; иначе говоря, $\varphi$ была бы константой и движение было бы стационарным. Это означает, что мы должны различать два типа движения: либо производная $\dot{\varphi}$ все время сохраняет один и тот же знак, либо $\varphi$ принимает одно значение, при котором $\dot{\varphi}=0$.

Как следует из (15), равенство $\dot{\varphi}=0$ имеет место, когда $k^{2}\left(c_{1}^{2}-c_{2}^{2}\right) \cos ^{2} \varphi+c_{1}^{2} \sin ^{2} \varphi=0$, а у этого уравнения решения есть только в случае $c_{1}^{2}-c_{2}^{2} \leqslant 0$. Если $c_{1}^{2}-c_{2}^{2}>0$, то при каждом значении $\varphi$ производная $\dot{\varphi}$ сохраняет один и тот же знак, а именно, знак константы $c_{1}$. Если же $c_{1}^{2}-c_{2}^{2}<0$ и $c_{1} \neq 0$, то существует один угол $\varphi_{1}\left(0<\varphi_{1}<\pi\right)$, для которого $\operatorname{tg} \varphi_{1}=$ $=k \operatorname{tg} \psi_{1}$ и $\cos \psi_{1}=-c_{1} / c_{2}$, и тогда $\dot{\varphi}=0$ при $\varphi= \pm \varphi_{1}$. В этом случае производная $\dot{\varphi}$ тоже всегда сохраняет один и тот же знак, причем он снова совпадает со знаком константы $c_{1}$, но угол $\varphi$ уже не может принимать любое значение. Он либо всегда принадлежит интервалу $-\varphi_{1}<\varphi<\varphi_{1}$, либо всегда лежит в интервале, его дополняющем $\left(\varphi_{1}<\varphi<2 \pi-\varphi_{1}\right)$. Впрочем, последний случай можно не рассматривать, так как, поменяв местами точки $P_{1}$ и $P_{2}$ (либо поменяв знак константы $c_{1}$ ), мы сведем его к предыдущему. Если теперь предположить, что $c_{1}>0$, что, очевидно, не накладывает на задачу никаких существенных ограничений, то мы получим следующее решение: при изменении $t$ от $-\infty$ до $+\infty$ угол $\varphi$ будет возрастать от $-\varphi_{1}$ до $\varphi_{1}$ и пройдет через положение $\varphi=0$. Если при этом $c_{2}<0$, то угол $\varphi_{1}$ будет острым, то есть положения $\varphi= \pm \pi / 2$ не достигаются; но при $c_{2}>0$ угол $\varphi$ проходит оба этих положения; значение $c_{2}=0$ несовместимо с нашим предположением.

В итоге у нас есть следующие основные случаи:

I: $c_{1}^{2}-c_{2}^{2}>0, c_{2} \neq 0$; у траектории $C(Q)$ бесконечно много точек возврата и точек перегиба;

IIa: $c_{1}^{2}-c_{2}^{2}<0, c_{1} c_{2}>0$; у траектории $C(Q)$ одна точка возврата и две точки перегиба;

IIb: $c_{1}^{2}-c_{2}^{2}<0, c_{1} c_{2}<0$; у траектории $C(Q)$ одна точка возврата и нет точек перегиба. 
6. Теперь обсудим исключенные нами ранее особые случаи. Случай $c_{1}=0$ будет граничным для варианта II. При этом $\varphi_{1}=\pi / 2$. Если $c_{2} \neq 0$, то мы, очевидно, можем считать, что $c_{2}>0$. Тогда угол $\varphi$ изменяется от $-\pi / 2$ до $\pi / 2$. У кривой $C(Q)$ есть одна точка возврата, а при $t \rightarrow-\infty$ и $t \rightarrow+\infty$ она асимптотически стремится к точке перегиба.

Если наряду с $c_{1}=0$ еще и $c_{2}=0$, то углы $\varphi$ и $\vartheta$ постоянны, а $u=0$. Система находится в состоянии покоя. При $c_{2}=0, c_{1} \neq 0$ тело $L_{1}$ покоится, а тело $L_{2}$ вращается с постоянной угловой скоростью. Когда $c_{1}^{2}-c_{2}^{2}=0$, то либо $\varphi_{1}=0$ при $c_{2}<0$, либо $\varphi_{1}=\pi$ при $c_{2}>0$; в первом случае угол $\varphi$ пробегает интервал $0<\varphi<2 \pi$, когда $t$ изменяется от $-\infty$ до $+\infty$; во втором случае - интервал $-\pi<\varphi<\pi$. Кривая $C(Q)$ имеет две точки перегиба и стремится к точке возврата с обеих сторон.

Заметим, что во всех случаях, где возникает параметр $\varphi_{1}$, мы можем подобрать начальные условия так, чтобы равенство $\varphi=\varphi_{1}$ выполнялось для любого значения $t$. Мы будем называть такое движение стационарным. Поскольку радиус кривизны траектории $C(Q)$ зависит только от угла $\varphi$, кривая $C(Q)$ в этом случае представляет собой окружность с радиусом $l\left|\operatorname{tg} \varphi_{1}\right|$ и, поскольку из (17) следует, что $\dot{\vartheta}=-c_{2} \cos \psi_{1}=c_{1}$, точка $Q$ равномерно вращается вокруг неподвижной точки $G$, причем прямая $P_{1} P_{2}$ всегда проходит через точку $G$ (рис. 3 ). Движение происходит в прямом $\left(u=k c_{2} \sin \psi_{1}>0\right)$ или обратном $(u<0)$ направлении. При $\varphi_{1}=\pi / 2$ траектория $C(Q)-$ это прямая; при $\varphi_{1}=0$ точка $Q$ неподвижна.

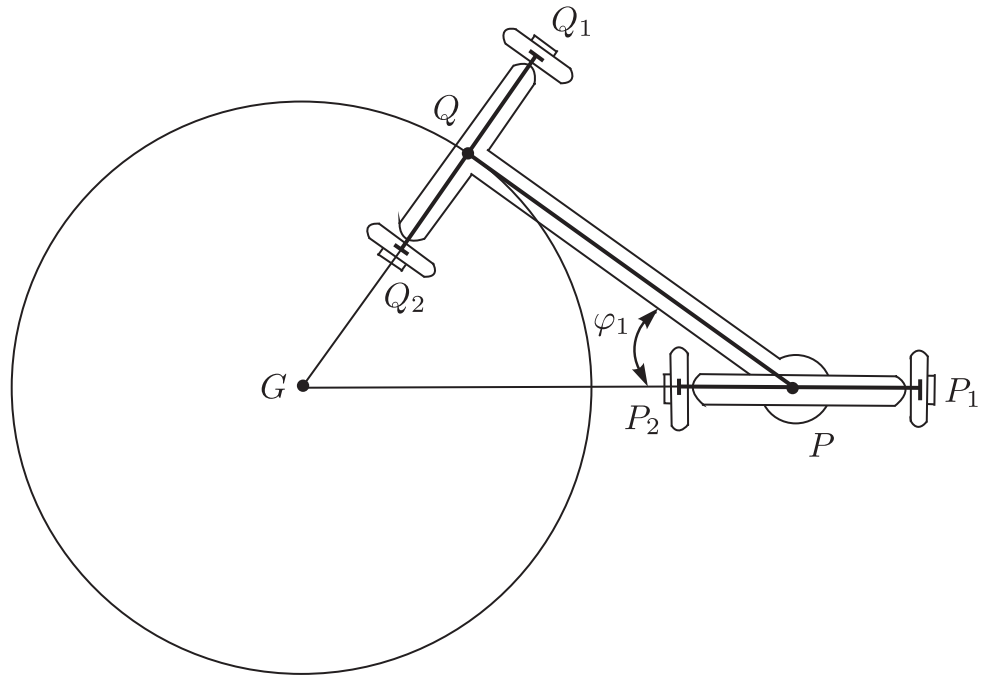

Рис. 3

7. Теперь вернемся к дифференциальному уравнению (19). Первое выражение, стоящее в числителе, положительно определено, так что его можно разложить в произведение двух сомножителей второй степени. При $k^{2} \leqslant 1,1-k^{2}=k_{1}^{2}$ получаем

$$
v^{4}+2\left(2 k^{2}-1\right) v^{2}+1=\left(v^{2}-2 k_{1} v+1\right)\left(v^{2}+2 k_{1} v+1\right),
$$

а при $k^{2} \geqslant 1, k^{2}-1=k_{2}^{2}$ получаем

$$
v^{4}+2\left(2 k^{2}-1\right) v^{2}+1=\left\{v^{2}+\left(k-k_{2}\right)^{2}\right\}\left\{v^{2}+\left(k+k_{2}\right)^{2}\right\} .
$$


Если $k^{2} \leqslant 1$, то возникает следующее разложение на простейшие дроби:

$$
\begin{aligned}
& \frac{\left(1+v^{2}\right)^{2}}{\left\{v^{4}+2\left(2 k^{2}-1\right) v^{2}+1\right\}\left\{\left(c_{1}-c_{2}\right) v^{2}+\left(c_{1}+c_{2}\right)\right\}}= \\
& \quad=\frac{A+B v}{v^{2}-2 k_{1} v+1}+\frac{A-B v}{v^{2}+2 k_{1} v+1}+\frac{C_{1}}{\left(c_{1}-c_{2}\right) v^{2}+\left(c_{1}+c_{2}\right)},
\end{aligned}
$$

где

$$
A=\frac{k_{1}^{2}\left(c_{1}-c_{2}\right)}{2\left(k_{1}^{2} c_{1}^{2}+k^{2} c_{2}^{2}\right)}, \quad B=\frac{k_{1} c_{2}}{2\left(k_{1}^{2} c_{1}^{2}+k^{2} c_{2}^{2}\right)}, \quad C_{1}=\frac{c_{2}^{2}}{k_{1}^{2} c_{1}^{2}+k^{2} c_{2}^{2}} .
$$

При $k^{2} \geqslant 1$ и $k_{2}^{2} c_{1}^{2}-k^{2} c_{2}^{2} \neq 0$ находим

$$
\begin{aligned}
& \frac{\left(1+v^{2}\right)^{2}}{\left\{v^{4}+2\left(2 k^{2}-1\right) v^{2}+1\right\}\left\{\left(c_{1}-c_{2}\right) v^{2}+\left(c_{1}+c_{2}\right)\right\}}= \\
& \quad=\frac{A_{1}}{v^{2}+\left(k-k_{2}\right)^{2}}+\frac{A_{2}}{v^{2}+\left(k+k_{2}\right)^{2}}+\frac{C_{2}}{\left(c_{1}-c_{2}\right) v^{2}+\left(c_{1}+c_{2}\right)}
\end{aligned}
$$

где

$$
A_{1}=\frac{k_{2}\left(k-k_{2}\right)}{2 k\left(k_{2} c_{1}+k c_{2}\right)}, \quad A_{2}=\frac{k_{2}\left(k+k_{2}\right)}{2 k\left(k_{2} c_{1}-k c_{2}\right)}, \quad C_{2}=\frac{c_{2}^{2}}{k^{2} c_{2}^{2}-k_{2}^{2} c_{1}^{2}} .
$$

В особом случае $k^{2} \geqslant 1, k_{2} c_{1}+k c_{2}=0$ разложение имеет вид

$$
\frac{A_{1}^{1}}{\left\{v^{2}+\left(k-k_{2}\right)^{2}\right\}^{2}}+\frac{A_{2}^{1}}{v^{2}+\left(k+k_{2}\right)^{2}}+\frac{C_{2}^{1}}{\left(c_{1}-c_{2}\right) v^{2}+\left(c_{1}+c_{2}\right)},
$$

где

$$
A_{1}^{1}=\frac{k_{2}\left(k-k_{2}\right)^{2}}{k\left(c_{1}-c_{2}\right)}, \quad A_{2}^{1}=\frac{\left(k+k_{2}\right)^{2}}{4 k^{2}\left(c_{1}-c_{2}\right)}, \quad C_{2}^{1}=\frac{\left(k-k_{2}\right)\left(3 k+k_{2}\right)}{4 k^{2}},
$$

и аналогичные формулы возникают в случае $k^{2}>1, k_{2} c_{1}-k c_{2}=0$.

8. Теперь, проинтегрировав (19) с помощью разложений $(20),(21)$ и (22), мы получим следующий результат:

$$
t=F(\varphi)+G(\varphi)
$$

где

$$
\begin{aligned}
F(\varphi) & =k k_{1}\left(k_{1}^{2} c_{1}^{2}+k^{2} c_{2}^{2}\right)^{-1}\left\{k_{1} c_{1} \varphi+\frac{1}{2} c_{2} \ln \frac{1-k_{1} \sin \psi}{1+k_{1} \sin \psi}\right\}, \quad k^{2} \leqslant 1, \\
F(\varphi) & =k k_{2}\left(k_{2}^{2} c_{1}^{2}-k^{2} c_{2}^{2}\right)^{-1}\left\{k_{2} c_{1} \varphi-c_{2} \operatorname{arctg}\left(k_{2} \sin \psi\right)\right\}, \quad k^{2} \geqslant 1, \quad k_{2}^{2} c_{1}^{2}-k^{2} c_{2}^{2} \neq 0, \\
F(\varphi) & =\left\{4 k\left(k-k_{2}\right)\left(c_{1}-c_{2}\right)\right\}^{-1} \times \\
& \times\left\{\left(2 k k_{2}+1\right) \varphi+\left(2 k k_{2}-1\right) \operatorname{arctg}\left(k_{2} \sin \psi\right)+2 k k_{2} \frac{\sin \psi}{k-k_{2} \cos \psi}\right\}, \\
k^{2} & >1, \quad k_{2} c_{1}+k c_{2}=0,
\end{aligned}
$$

и аналогичная формула при $k^{2}>1, k_{2} c_{1}-k c_{2}=0$. 
Кроме того, у нас

$$
\begin{aligned}
& G(\varphi)=\frac{2 k}{c} C \operatorname{arctg}\left(\frac{c}{c_{1}+c_{2}} \operatorname{tg} \frac{1}{2} \psi\right), \quad 0<c_{1}^{2}-c_{2}^{2}=c^{2}, \\
& G(\varphi)=\frac{k}{c_{1}} C \operatorname{tg} \frac{1}{2} \psi, \quad c_{1}=c_{2}, \\
& G(\varphi)=\frac{-k}{c_{1}} C \operatorname{ctg} \frac{1}{2} \psi, \quad c_{1}=-c_{2}, \\
& G(\varphi)=\frac{k}{c_{1}+c_{2}} C \ln \frac{\sin \frac{1}{2}\left(\psi_{1}+\psi\right)}{\sin \frac{1}{2}\left(\psi_{1}-\psi\right)}, \quad c_{1}^{2}-c_{2}^{2}<0, \quad \cos \psi_{1}=\frac{-c_{1}}{c_{2}} .
\end{aligned}
$$

В формулах (27)-(30) необходимо заменять $C$ на $C_{1}, C_{2}$ или $C_{2}^{\prime}$ в зависимости от того, $k^{2} \leqslant 1, k^{2} \geqslant 1$ и т. д.

Кроме того, мы предполагали, что $\varphi=0$ при $t=0$, за исключением случая (29), когда равенство $\varphi=0$ не выполняется ни для одного конечного значения $t$, и мы будем предполагать, что $\varphi=\pi$ при $t=0$.

Формулы (23)-(30) позволяют определить взаимосвязь между $\varphi$ и $t$ во всех случаях.

Функция $F(\varphi)$ конечна при любом значении $\varphi$, а из $(27)$ следует, что то же самое верно и для $G(\varphi)$. В (28) при $t \rightarrow \infty$ выполняется $\varphi \rightarrow \pi$; в (29) $\varphi \rightarrow 0$, а в (30) $\varphi \rightarrow \varphi_{1}$.

Когда $0<c_{1}^{2}-c_{2}^{2}$, тело $L_{2}$ совершает полные обороты. Время, необходимое для поворота от $\varphi=0$ до $\varphi=\pi / 2$, составляет $t_{1}=F(\pi / 2)+G(\pi / 2)$, а время поворота от $\varphi=0$ до $\varphi=\pi$ равно $t_{2}=F(\pi)+G(\pi)$; здесь $t_{2}$ - это время движения по кривой $C(Q)$ от одной точки возврата до другой, а $t_{1}$ либо, соответственно, $\left(t_{2}-t_{1}\right)$ - время движения между точкой возврата и точкой перегиба.

9. Поскольку $\vartheta+\varphi=c_{1} t$, формула (23) также позволяет нам установить соотношение между $\vartheta$ и $\varphi$. Очевидно, что разность $\left(c_{1} t_{1}-\pi / 2\right)$ задает угол между касательными, проходящими через точку возврата и точку перегиба, а $\left(c_{1} t_{2}-\pi\right)$ - угол между касательными, проходящими через две соседние точки возврата.

$\mathrm{У}$ нас $\dot{x}=u \cos \vartheta$, откуда

$$
\frac{d x}{d \varphi}=\frac{l k c_{2} \sin \varphi \cos \vartheta}{c_{1} \sqrt{\sin ^{2} \varphi+k^{2} \cos ^{2} \varphi}+k c_{2} \cos \varphi},
$$

что позволяет выразить $d x / d \varphi$ через $\varphi$. Таким образом, мы сможем определить $x$ и $y$ через $\varphi$ с помощью квадратур и найти тем самым траекторию $C(Q)$. Впрочем, данные квадратуры, судя по всему, не допускают запись в явной форме.

10. Координаты полюса $x_{G}$ и $y_{G}$ имеют вид

$$
x_{G}=x+l \operatorname{tg} \varphi \sin \vartheta, \quad y_{G}=y-l \operatorname{tg} \varphi \cos \vartheta,
$$

откуда следует

$$
\dot{x}_{G}=l \frac{\dot{\varphi} \sin \vartheta}{\cos ^{2} \varphi}, \quad \dot{y}_{G}=-l \frac{\dot{\varphi} \cos \vartheta}{\cos ^{2} \varphi},
$$

так что

$$
\frac{d x_{G}}{d \varphi}=l \frac{\sin \vartheta}{\cos ^{2} \varphi}, \quad \frac{d y_{G}}{d \varphi}=-l \frac{\cos \vartheta}{\cos ^{2} \varphi}
$$


и положение точки $G$, а значит, и неподвижная полярная кривая, описывающая движение тела $L_{1}$, допускают определение в квадратурах; впрочем, и эти квадратуры в общем случае не так-то просто исследовать.

11. Рассмотрим на отрезке $Q P$ точку $A$, для которой $Q A=k l$. Координаты точки $A$ имеют вид

$$
x_{A}=x+k l \cos \vartheta, \quad y_{A}=y+k l \sin \vartheta,
$$

тогда

$$
\left\{\begin{array}{l}
\dot{x}_{A}=\dot{x}-k l \sin \vartheta \cdot \dot{\vartheta}=u \cos \vartheta+k l c_{2} \sin \vartheta \cos \psi=l k c_{2} \sin (\vartheta+\psi), \\
\dot{y}_{A}=-l k c_{2} \cos (\vartheta+\psi) .
\end{array}\right.
$$

Иначе говоря, скорость точки $A$ во время движения постоянна по величине и равна $l k c_{2}$. Радиус кривизны траектории точки $A$ принимает переменное значение $l k c_{2} /(\dot{\vartheta}+\dot{\psi})$.

12. В завершение укажем, что при $k=1$ движение будет очень простым; тогда $l^{2}=$ $=\rho_{1}^{2}+a^{2}$, что, очевидно, вполне возможно. В этом случае траекторию можно полностью определить в элементарных функциях.

Дифференциальное уравнение для $\varphi$ выглядит так:

$$
\dot{\varphi}=c_{1}+c_{2} \cos \varphi
$$

Вспомогательный угол $\psi$ совпадает с $\varphi$, функция $F(\varphi)$ тождественно равна нулю и $C=1$.

При

$$
u=l c_{2} \sin \varphi, \quad \dot{\vartheta}=-c_{2} \cos \varphi, \quad \vartheta+\varphi=c_{1} t
$$

получим

$$
\dot{x}=u \cos \vartheta=l c_{2} \sin \varphi \cos \vartheta=l c_{2}\left(\sin c_{1} t-\sin \vartheta \cos \varphi\right)=l c_{2} \sin c_{1} t+l \sin \vartheta \cdot \dot{\vartheta},
$$

поэтому

$$
x=-l \frac{c_{2}}{c_{1}} \cos c_{1} t-l \cos \vartheta+b, \quad b=l \frac{c_{1}+c_{2}}{c_{1}}
$$

и

$$
y=-l \frac{c_{2}}{c_{1}} \sin c_{1} t-l \sin \vartheta
$$

Откуда следует

$$
(x-b)^{2}+y^{2}=\frac{l^{2}}{c_{1}^{2}}\left(c_{1}^{2}+c_{2}^{2}+2 c_{1} c_{2} \cos \varphi\right)
$$

что позволяет нам сделать следующий вывод: точка $Q$ при движении остается внутри кольца, ограниченного концентрическими окружностями $K_{1}$ и $K_{2}$ с центром $M(b, 0)$ и радиусами

$$
R_{1}=l\left|\frac{c_{1}+c_{2}}{c_{1}}\right| \quad \text { и } \quad R_{2}=l\left|\frac{c_{1}-c_{2}}{c_{1}}\right|,
$$

причем одна из окружностей проходит через начало координат $O$. Справедливо равенство $R_{1}+R_{2}=2 l$. Возможные точки возврата кривой $C(Q)$ лежат на окружностях $K_{1}$ и $K_{2}$ поочередно. Точки перегиба $(\cos \varphi=0)$ лежат на третьей концентрической окружности $K_{3}$, радиус которой равен

$$
R_{3}=l\left(\frac{c_{1}^{2}+c_{2}^{2}}{c_{1}^{2}}\right)^{1 / 2}
$$


В этом случае точка $A$ совпадает с точкой $P$; учитывая, что $\vartheta+\psi=\vartheta+\varphi=c_{1} t$, находим для этой точки

$$
\dot{x}_{p}=l c_{2} \sin c_{1} t, \quad \dot{y}_{p}=-l c_{2} \cos c_{1} t
$$

и, следовательно,

$$
x_{p}=-l \frac{c_{2}}{c_{1}} \cos c_{1} t+b, \quad y_{p}=-l \frac{c_{2}}{c_{1}} \sin c_{1} t .
$$

Таким образом, траектория точки $P$ - это концентрическая окружность $K_{4}$ с радиусом $R_{4}=l\left|c_{2} / c_{1}\right|$, причем точка $P$ пробегает ее с постоянной угловой скоростью $c_{1}$.

Поэтому движение отрезка $P Q$ происходит так (рис. 4), что точка $P$ описывает окружность, тогда как скорость точки $Q$ направлена вдоль прямой $P Q$. Это означает, что точка $Q$ пробегает кривую, известную как круговая трактриса [2]. Главная окружность $K_{0}$ данной кривой имеет общий центр с окружностью $K_{4}$ и радиус $R_{0}$, где $R_{0}^{2}=R_{4}^{2}-l^{2}$. Возникают два основных случая. Когда $R_{0}^{2}<0$, окружность $K_{0}$ - мнимая; кривая $C(Q)$ состоит из конгруэнтных дуг, содержащих по одной точке перегиба и пересекающих друг друга в точках возврата, которые располагаются поочередно на окружностях $K_{1}$ и $K_{2}$ (рис. 5 ). В общем случае эта кривая трансцендентна, но может оказаться и алгебраической (рис. 6). Если же $R_{0}^{2}>0$, то окружность $K_{0}$ действительна; у кривой есть одна точка возврата; кривая асимптотически стремится к $K_{0}$, совершая бесконечное число оборотов; при этом траектория $C(Q)$ может располагаться как внутри (рис. 7), так и снаружи (рис. 8) главной окружности. Переходный случай возникает при $R_{0}^{2}=0$. Тогда окружность $K_{0}-$ нулевая, а точка $M$ - асимптотическая точка исследуемой кривой. Для нашего движения $R_{4}^{2}=$ $=l^{2} c_{2}^{2} / c_{1}^{2}$, так что $R_{0}^{2}=l^{2}\left(c_{2}^{2}-c_{1}^{2}\right) / c_{1}^{2}$. Если $c_{1}^{2}-c_{2}^{2}=c^{2}>0$, то главная окружность будет мнимой. Теперь из (23) следует, что

$$
t=\frac{2}{c} \operatorname{arctg}\left(\frac{c}{c_{1}+c_{2}} \operatorname{tg} \frac{1}{2} \varphi\right)
$$

или

$$
\operatorname{tg} \frac{1}{2} \varphi=\frac{c_{1}+c_{2}}{c} \operatorname{tg} \frac{1}{2} c t
$$

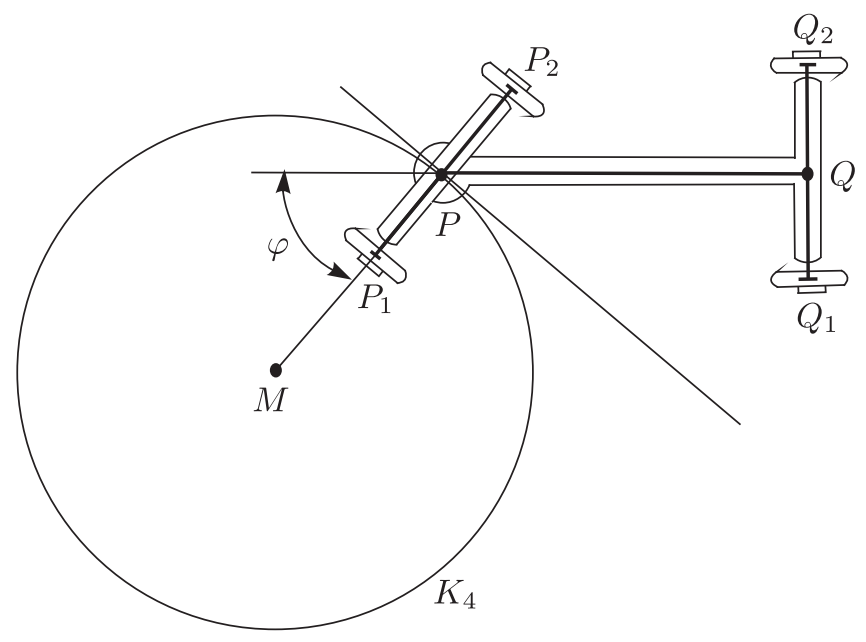

Рис. 4 


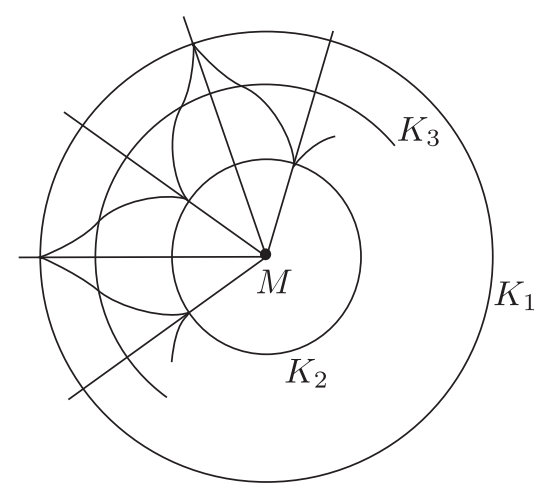

Рис. 5

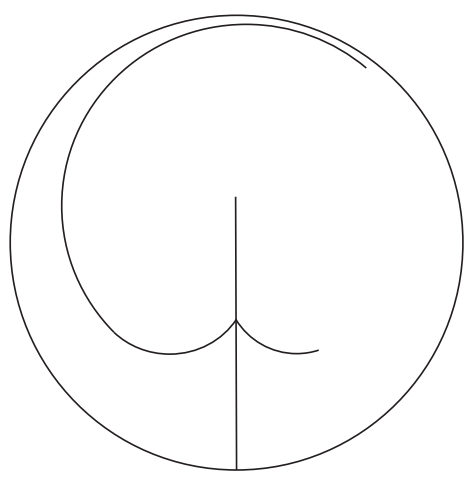

Рис. 7

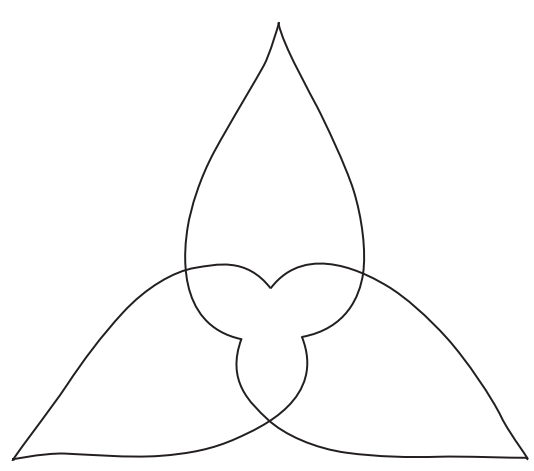

Рис. 6

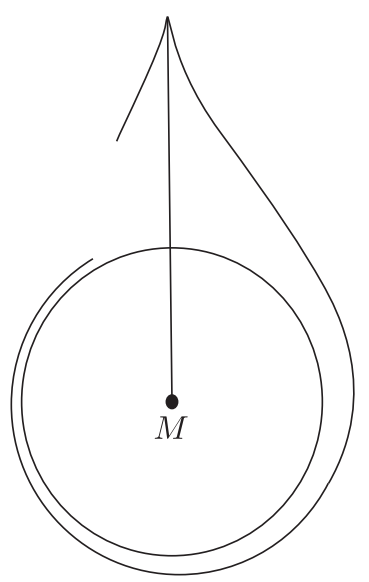

Рис. 8

Точки возврата кривой $C(Q)$ лежат на окружности $K_{1}$ при $c t=0,2 \pi, 4 \pi, \ldots$ и на окружности $K_{2}$ при $c t=\pi, 3 \pi, \ldots$, а точки перегиба лежат на окружности $K_{3}$ при $c t=\pi / 2,3 \pi / 2, \ldots$. Время движения от $K_{1}$ до $K_{3}$ составляет $t_{1}=(2 / c) \operatorname{arctg}\left\{c /\left(c_{1}+c_{2}\right)\right\}$, при этом угол $\vartheta$ возрастает на $\pi / c-c_{1} t_{1}$; движение от $K_{1}$ до $K_{2}$ занимает время $t_{2}=\pi / c$, а угол $\vartheta$ становится больше на $\pi\left(c-c_{1}\right) / c$.

В случае $c_{1}^{2}-c_{2}^{2}<0$ главная окружность действительна; при $t \rightarrow \infty$ модель стремится к стационарному движению вперед, при $t \rightarrow-\infty-$ к стационарному движению назад, которое выглядит неустойчивым. Из (23) следует, что

$$
t=\frac{1}{c_{1}+c_{2}} \ln \frac{\sin \frac{1}{2}\left(\varphi_{1}+\varphi\right)}{\sin \frac{1}{2}\left(\varphi_{1}-\varphi\right)}, \quad \cos \varphi_{1}=\frac{-c_{1}}{c_{2}}
$$

то есть

$$
\operatorname{tg} \frac{1}{2} \varphi=\operatorname{tg} \frac{1}{2} \varphi_{1} \cdot \operatorname{th} \frac{c_{1}+c_{2}}{2} t
$$

Имеем

$$
\begin{gathered}
R_{1}^{2}=\frac{l^{2}}{c_{1}^{2}}\left(c_{1}+c_{2}\right)^{2}, \quad R_{2}^{2}=\frac{l^{2}}{c_{1}^{2}}\left(c_{1}-c_{2}\right)^{2}, \quad R_{3}^{2}=\frac{l^{2}}{c_{1}^{2}}\left(c_{1}^{2}+c_{2}^{2}\right), \\
R_{0}^{2}=\frac{l^{2}}{c_{1}^{2}}\left(c_{2}^{2}-c_{1}^{2}\right)=l^{2} \operatorname{tg}^{2} \varphi_{1}
\end{gathered}
$$


при этом из (35) следует

$$
M Q^{2}=\frac{l^{2}}{c_{1}^{2}}\left(c_{1}^{2}+c_{2}^{2}+2 c_{1} c_{2} \cos \varphi\right) .
$$

Если предположить, что $c_{1}+c_{2}>0$, то $\varphi$ пробегает интервал $-\varphi_{1}<\varphi<\varphi_{1}$. При $c_{1} c_{2}>0$ угол $\varphi_{1}$ - тупой, и у нас $R_{1}^{2}>R_{3}^{2}>R_{0}^{2}>R_{2}^{2}$, причем $R_{0}^{2}<M Q^{2} \leqslant R_{1}^{2}$. Таким образом, кривая $C(Q)$ лежит снаружи главной окружности $K_{0}$, у нее есть одна точка возврата на окружности $K_{1}$ и две точки перегиба на окружности $K_{3}$. Если, наоборот, $c_{1} c_{2}<0$, то $R_{2}^{2}>R_{3}^{2}>R_{0}^{2}>R_{1}^{2}$ и $R_{1}^{2} \leqslant M Q^{2}<R_{0}^{2}$, то есть трактриса лежит внутри окружности $K_{0}$, у нее есть одна точка возврата на окружности $K_{1}$ и нет точек перегиба. Аналогичный результат получаем при $c_{1}+c_{2}<0$.

В особом случае $c_{1}=c_{2} \neq 0$ имеем $\operatorname{tg}(\varphi / 2)=c_{1} t, R_{0}=R_{2}=0$. У кривой есть одна точка возврата на окружности $K_{1}$, две точки перегиба на окружности $K_{3}$; кривая асимптотически стремится к центру $M$.

При $c_{1}=0, c_{2} \neq 0$ радиусы окружностей $K_{i}$ будут бесконечны; тогда у нас $\operatorname{tg}(\varphi / 2)=$ $=\operatorname{th}\left(c_{2} t / 2\right), \sin \varphi=\operatorname{th} c_{2} t, \vartheta=-\varphi, u=l c_{2} \sin \varphi$ и в силу

$$
\dot{x}=\frac{l c_{2} \operatorname{sh} c_{2} t}{\operatorname{ch}^{2} c_{2} t}, \quad \dot{y}=-l c_{2} \operatorname{th}^{2} c_{2} t
$$

получаем

$$
x=-\frac{l}{\operatorname{ch} c_{2} t}+l, \quad y=l\left(\operatorname{th} c_{2} t-c_{2} t\right),
$$

так что кривая $C(Q)$ оказывается обыкновенной трактрисой.

\section{References}

[1] Carathéodory C. Der Schlitten, Z. Angew. Math. Mech., 1933, vol.13, no. 2, pp. 71-76.

[2] Loria G. Spezielle algebraische und transzendente ebene Kurven: Theorie und Geschichte: Vol. 2. Die transzendenten und die abgeleiteten Kurven, Leipzig: Teubner, 1911, pp. 195-200.

\section{Die Bewegung eines einfachen Wagenmodells}

O. Bottema

Citation: Rus. J. Nonlin. Dyn., 2015, vol. 11, no. 3, pp. 621-632 (Russian) 